\title{
Hadronic physics at KLOE
}

\author{
Roberto Versaci on behalf of the KLOE collaboration ${ }^{1}$ \\ Dipartimento di Energetica dell'Università “Sapienza”, Roma, Italy \\ Laboratori Nazionali di Frascati dell'INFN, Frascati, Italy
}

\begin{abstract}
New KLOE results on scalar mesons, $\gamma \gamma$ physics and $\eta$ physics are presented.
Keywords: Scalar mesons, $\gamma \gamma$ physics, $\eta$ meson.

PACS: 13.25.Es, 13.25.Jx, 13.66.Bc, 14.40.Aq, 14.40.Cs
\end{abstract}

\section{SCALAR PHYSICS}

The structure of the scalars below $1 \mathrm{GeV}$ needs to be further clarified. Several models have been proposed to describe them (e.g. $q \bar{q}$, four quarks, $K \bar{K}$ molecules, etc.). The decay of the scalars into two pseudoscalars $\left(S \rightarrow P P^{\prime}\right)$ can be used to investigate their nature because the branching ratios and the invariant mass of the two pseudoscalars are sensitive to the scalar structure.

$\phi \rightarrow a_{0}(980) \gamma \rightarrow \eta \pi^{0} \gamma$ decay [1] $]$. For this measurement about $400 \mathrm{pb}^{-1}$ of KLOE collected data have been used. The analysis has been performed for two different $\eta$ final states, i.e. $\eta \rightarrow \gamma \gamma$ and $\eta \rightarrow \pi^{+} \pi^{-} \pi^{0}$. A kinematic fit has been performed imposing the four momentum conservation, the photon velocity and the invariant masses of both $\eta$ and $\pi^{0}$. The $\eta \pi^{0}$ invariant mass distribution has been fitted with the "no-structure"[2] and the "kaon loop" [3] models after background subtraction. The results of the fit are shown in table 1, It is interesting to note that both models give a large coupling of the $a_{0}(980)$ with the $\phi$ meson, indicating a sizable strange quark content in the $a_{0}(980)$. The branching ratio obtained for the two different decay chains are in agreement: $B R\left(\phi \rightarrow \eta \pi^{0} \gamma\right)=\left(7.01 \pm 0.10_{\text {stat. }} \pm 0.20_{\text {syst. }}\right) \times 10^{-5}$ for the $\eta \rightarrow \gamma \gamma$ final state and $B R\left(\phi \rightarrow \eta \pi^{0} \gamma\right)=\left(7.12 \pm 0.13_{\text {stat. }} \pm 0.22_{\text {syst }}\right) \times 10^{-5}$ for the $\eta \rightarrow \pi^{+} \pi^{-} \pi^{0}$ final state.

$\phi \rightarrow K^{0} \bar{K}^{0} \gamma$ decay [4]. This decay allegedly proceeds through the intermediate $f_{0}(980)(\mathrm{I}=0)$ and $a_{0}(980)(\mathrm{I}=1)$ scalar mesons: $\phi \rightarrow\left(f_{0}+a_{0}\right) \gamma \rightarrow K^{0} \bar{K}^{0} \gamma$. The kaon pair is produced in a $J^{P C}=0^{++}$state, so the two kaons are both $K_{S}$ or $K_{L}$. We have searched for a final state with a $K_{S} K_{S}$, with both $K_{S}$ ' decaying to $\pi^{+} \pi^{-}$. This request

\footnotetext{
${ }^{1}$ F. Ambrosino, A. Antonelli, M. Antonelli, F. Archilli, P. Beltrame, G. Bencivenni, S. Bertolucci, C. Bini, C. Bloise, S. Bocchetta, F. Bossi, P. Branchini, G. Capon, T. Capussela, F. Ceradini, P. Ciambrone, E. De Lucia, A. De Santis, P. De Simone, G. De Zorzi, A. Denig, A. Di Domenico, C. Di Donato, B. Di Micco, M. Dreucci, G. Felici, S. Fiore, P. Franzini, C. Gatti, P. Gauzzi, S. Giovannella, E. Graziani, G. Lanfranchi, J. Lee-Franzini, M. Martini, P. Massarotti, S. Meola, S. Miscetti, M. Moulson, S. Müller, F. Murtas, M. Napolitano, F. Nguyen, M. Palutan, E. Pasqualucci, A. Passeri, V. Patera, P. Santangelo, B. Sciascia, T. Spadaro, M. Testa, L. Tortora, P. Valente, G. Venanzoni, R.Versaci, G. Xu
} 
TABLE 1. Output of the fit to the $\eta \pi^{0}$ invariant mass with two different models: kaon loop and no structure.

\begin{tabular}{lll}
\hline & Kaon loop & No structure \\
\hline$M_{a_{0}}[\mathrm{MeV}]$ & $982.5 \pm 1.6 \pm 1.1$ & 982.5 (Fixed) \\
$g_{a_{0} K^{+} K^{-}}[\mathrm{GeV}]$ & $2.15 \pm 0.06 \pm 0.06$ & $2.01 \pm 0.07 \pm 0.28$ \\
$g_{a_{0} \eta \pi^{0}}[\mathrm{GeV}]$ & $2.82 \pm 0.03 \pm 0.04$ & $2.46 \pm 0.08 \pm 0.11$ \\
$g_{\phi a_{0} \gamma}\left[\mathrm{GeV}^{-1}\right]$ & $1.58 \pm 0.10 \pm 0.16^{*}$ & $1.83 \pm 0.03 \pm 0.08$ \\
$\mathrm{BR}(\phi \rightarrow \rho \pi \rightarrow \eta \pi \gamma)$ & $(0.92 \pm 0.40 \pm 0.1 \overline{5}) \cdot 10^{-6}$ & $(0.05 \pm 4 \pm 0.07) \cdot 10^{-6}$ \\
$\mathrm{BR}(\eta \rightarrow \gamma \gamma) / \mathrm{BR}\left(\eta \rightarrow \pi^{+} \pi^{-} \pi^{0}\right)$ & $1.70 \pm 0.04 \pm 0.03$ & $1.70 \pm 0.03 \pm 0.01$ \\
$\chi^{2}$ probability & 0.104 & 0.309 \\
\hline
\end{tabular}

* Not a free parameter of the fit in this model. Calculated from other fit outputs.

reduces the probability of observation to $\sim 22 \%$, but selects a class of event with a clear signature: four tracks and a low energy photon coming from the interaction point. In this analysis the whole KLOE dataset, $\sim 2.2 \mathrm{fb}^{-1}$, has been used. At the end of which we have observed 5 events in the data, while we were expecting $3.2 \pm 0.7$ background events from MC. A Cousin-Feldman approach has been used [5] and a 90\% confidence level upper limit on the branching ratio has been obtained: $B R\left(\phi \rightarrow K^{0} \bar{K}^{0} \gamma\right)<1.9 \times 10^{-8}$. This measurement excludes some of the theoretical predictions and is in agreement with expectations from other KLOE measurements (see figure 11 left, reference [4] and references therein).

\section{2. $\gamma \gamma$ PHYSICS}

KLOE has been making a pilot study for the search $\gamma \gamma \rightarrow \sigma(600) \rightarrow \pi^{0} \pi^{0}$ using 11 $\mathrm{pb}^{-1}$ from the $240 \mathrm{pb}^{-1}$ taken at $\sqrt{s}=1000 \mathrm{MeV}$ [6]. At this energy the background
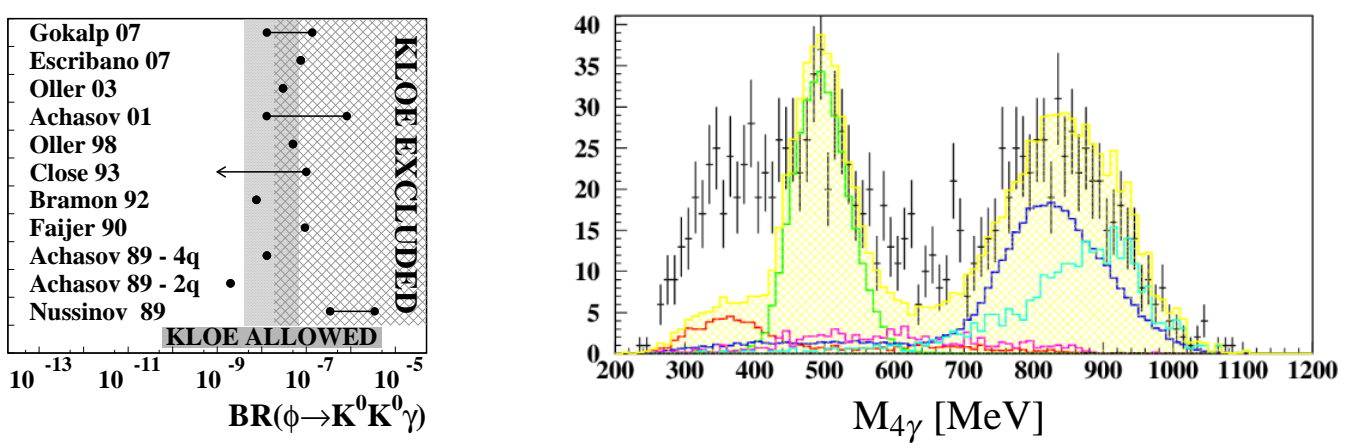

FIGURE 1. Left: comparison between theoretical predictions for the $\phi \rightarrow K^{0} \bar{K}^{0} \gamma$ branching ratio and KLOE upper limit. The grey area represents the expected range for the branching ratio using other KLOE results on scalar mesons. Right: search for $\gamma \gamma \rightarrow \sigma(600) \rightarrow \pi^{0} \pi^{0}$; fit to the invariant mass of the four photons, $\mathrm{M}_{4 \gamma}$, with MC shapes of expected source of background. Dots: data; atched yellow: total background; red: $\phi \rightarrow \eta \gamma \rightarrow \pi^{0} \pi^{0} \pi^{0} \gamma$; blue: $e^{+} e^{-} \rightarrow \omega \pi^{0} \rightarrow \pi^{0} \pi^{0} \gamma$; green: $\phi \rightarrow K_{S} K_{L}$; cyan: $\phi \rightarrow f_{0} \gamma$; magenta: $e^{+} e^{-} \rightarrow \gamma \gamma$. 
TABLE 2. Output of the fit imposing or not the gluonium content to be zero.

\begin{tabular}{lll}
\hline & Gluonium content forced to be zero & Gluonium content free \\
\hline$Z_{G}^{2}$ & fixed 0 & $0.115 \pm 0.036$ \\
$\phi_{P}$ & $(41.4 \pm 0.5)^{\circ}$ & $(40.4 \pm 0.6)^{\circ}$ \\
$Z_{q}$ & $0.93 \pm 0.02$ & $0.94 \pm 0.03$ \\
$Z_{s}$ & $0.82 \pm 0.05$ & $0.83 \pm 0.05$ \\
$\phi_{V}$ & $(3.34 \pm 0.09)^{\circ}$ & $(3.32 \pm 0.09)^{\circ}$ \\
$m_{s} / \bar{m}$ & $1.24 \pm 0.07$ & $1.24 \pm 0.07$ \\
$\chi^{2} /$ dof & $14.7 / 4$ & $4.6 / 3$ \\
$\mathrm{P}\left(\chi^{2}\right)$ & 0.005 & 0.20 \\
\hline
\end{tabular}

from $\phi$ decays is very small. We have performed a fit to the four photons invariant mass $\left(\mathrm{M}_{4 \gamma}\right)$, using the shapes of the known sources, see figure 1, right. The result of the fit is very poor $\chi^{2} /$ dof $=441 / 94$, showing an excess of events in the expected $\sigma(600)$ region, compared to what expected from MC, therefore pointing towards a search for the signal in the $240 \mathrm{pb}^{-1}$.

\section{PSEUDOSCALAR PHYSICS}

The $\phi$ meson decays about $1.3 \%$ of times into $\eta \gamma$, this implies DA $\Phi$ NE is an $\eta$-factory. KLOE has collected one of the largest sample of $\eta$ mesons in the world, about $10^{8}$.

$\eta-\eta^{\prime}$ mixing and $\eta^{\prime}$ gluonium content [7]. The KLOE paper on $\eta-\eta^{\prime}$ mixing [7], suggesting for a $3 \sigma$ evidence of gluonium content in the $\eta^{\prime}$ meson, has triggered a large amount of discussion among theoreticians. Therefore we have decided to perform a new and more detailed study of this topic. We have considered $\eta$ and $\eta^{\prime}$ in the quark mixing base as described in [8] $\left(\left|\eta^{\prime}>=X_{\eta^{\prime}}\right| q \bar{q}>+Y_{\eta^{\prime}}\left|s \bar{s}>+Z_{G}\right| G>\right)$. The new fit we have performed has more constraints thus allowing an independent determination of more free parameters. We use the BR values from PDG 2008 [9] and the new KLOE results on the $\omega$ meson [10]. The fit has been performed both imposing the gluonium content to be zero or allowing it free. The results are shown in table 2, gluonium content of the $\eta^{\prime}$ is confirmed at $3 \sigma$ level.

$\eta$ decays into four charged particles [11]. KLOE has started to study the decays of the $\eta$ into four charged particles, using $1.7 \mathrm{fb}^{-1}$ of data. This decay is interesting because it allows us to probe the $\eta$ internal structure exploiting the conversion of the virtual photon into a lepton pair [12]. It is also interesting because a non-CKM CP violating mechanism has been suggest to be present in this decay[13], and should manifest as an angular asymmetry $A_{\phi}$, between the pion and the electron decay planes in the $\eta$ rest frame. After background rejection a fit of the sidebands of the four tracks invariant distribution has been performed to obtain the background scale factors. Most of the background is due to $\phi$ decays, but there is still a non-negligible contribution from continuum events. Signal events have been counted in the $\eta$ mass region, giving $B R(\eta \rightarrow$ 

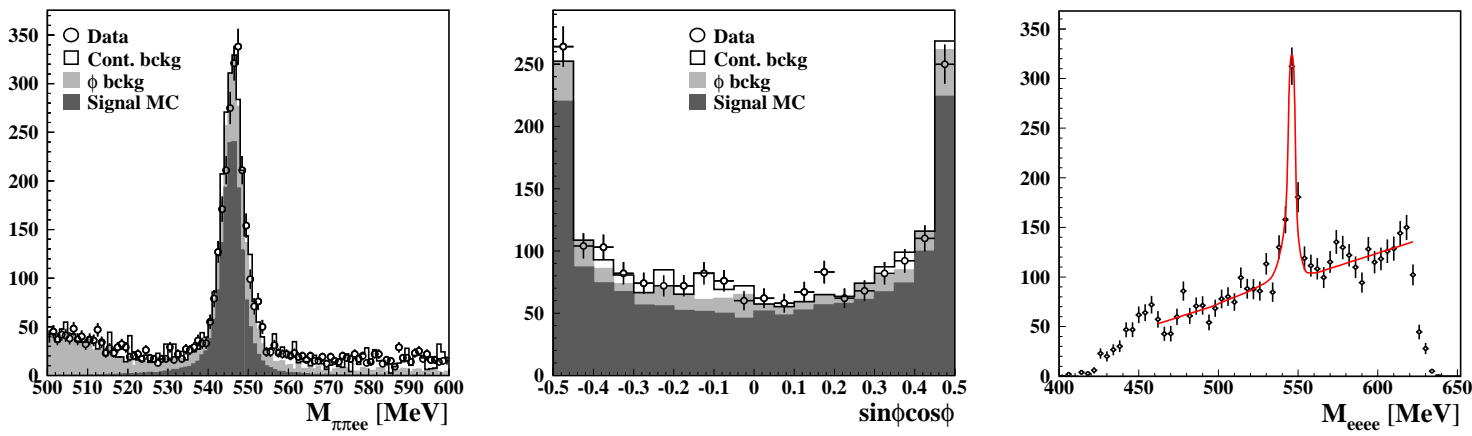

FIGURE 2. Left and center: $\eta \rightarrow \pi^{+} \pi^{-} e^{+} e^{-}$analysis; $\pi^{+} \pi^{-} e^{+} e^{-}$invariant mass and angular asymmetry distributions. Dots: data. The black histogram is the expected distribution, i.e. signal MC (dark grey), $\phi$ background (light grey) and continuum background (white). Right: $\eta \rightarrow e^{+} e^{-} e^{+} e^{-}$analysis; fit of the four electron invariant mass, $\mathrm{M}_{e e e e}$.

$\pi \pi e e)=\left(26.8 \pm 0.9_{\text {Stat. }} \pm 0.7_{\text {Syst }}\right) \times 10^{-5}$ and $A_{\phi}=\left(-0.6 \pm 2.5_{\text {Stat. }} \pm 1.8_{\text {Syst. }}\right) \times 10^{-2}$ [11], see figure 2]left and center.

More recently KLOE has started studying the $\eta \rightarrow e^{+} e^{-} e^{+} e^{-}$decay. This decay, together with the $\eta \rightarrow \mu^{+} \mu^{-} e^{+} e^{-}$, is interesting for the $\eta$ meson form factor because there are only leptons in the final state. The analysis strategy is similar to the $\pi \pi e e$ one. Most of the background comes from continuum events and a small contribution is due to $\phi$ decays. The latter is subtracted from data using the MC shape. The number of events is obtained fitting the data distribution of the 4 electron invariant mass, $\mathbf{M}_{e e e e}$, with signal and background shapes (figure 2, right). From the fit we obtain 413 events. This constitutes the first observation of this decay.

\section{REFERENCES}

1. F. Ambrosino, et al. (2009), 0904.2539.

2. G. Isidori, L. Maiani, M. Nicolaci, and S. Pacetti, JHEP 05, 049 (2006), hep-ph/ 0603241.

3. N. N. Achasov, and A. V. Kiselev, Phys. Rev. D68, 014006 (2003), hep-ph/ 0212153

4. F. Ambrosino, et al. (2009), 0903.4115.

5. G. J. Feldman, and R. D. Cousins, Phys. Rev. D57, 3873-3889 (1998), physics/9711021.

6. F. Ambrosino, et al., Nuovo Cim. 31C, 415 (2008).

7. F. Ambrosino, et al., Phys. Lett. B648, 267-273 (2007), hep-ex/0612029.

8. J. L. Rosner, Phys. Rev. D27, 1101 (1983).

9. C. Amsler, et al., Phys. Lett. B667, 1 (2008).

10. F. Ambrosino, et al., Phys. Lett. B669, 223-228 (2008), 0807.4909

11. F. Ambrosino, et al., Phys. Lett. B675, 283-288 (2009), 0812 . 4830.

12. L. G. Landsberg, Phys. Rept. 128, 301-376 (1985).

13. D.-N. Gao, Mod. Phys. Lett. A17, 1583-1588 (2002), hep-ph/0202002. 